\title{
On The Extinction Characteristics of Terahertz Wave in Fog
}

\author{
Qinghua Zhang ${ }^{1, a *}$ Hongxia Wang ${ }^{1, b}$ Jin Ma ${ }^{1, c}$ \\ ${ }^{1}$ Xi'an Research Inst. of Hi-Tech, Hongqing Town, Xi'an 710025, P.R. China \\ a15353652303@163.com, ${ }^{b}$ redlightw@163.com, ${ }^{c}$ majin_yx@163.com
}

\section{Keywords: Terahertz wave; Fog; Mie theory; extinction coefficient}

\begin{abstract}
In this paper, according to Mie theory and the particle size distribution of fog droplets, the total absorption and scattering of group particle were analyzed, the extinction parameters and attenuation rate of the Terahertz wave were calculated in radiation fog and advection fog through Matlab programs, and the relationship between the visibility and coefficients of scattering, absorption and extinction were obtained. The extinction and attenuation of Terahertz waves with different wavelengths were compared and analyzed in two different fogs. The result shows that the extinction parameters will drop sharply when the visibility increases; the lower the frequency of Terahertz waves is, the smaller the extinction coefficients will be; and the extinction and attenuation of Terahertz waves in advection fog is stronger than that of Terahertz waves in radiation fog because of the larger fog droplet size.
\end{abstract}

\section{Introduction}

Terahertz wave (THz wave) refers to the electromagnetic wave with frequencies between $0.1 \sim 10 \mathrm{THz}$ (wavelength between $30 \mu \mathrm{m} \sim 3 \mathrm{~mm}$ ), which is between microwaves and infrared on electromagnetic spectrum. THz wave has many superior characteristics ${ }^{[1]}$ because of its special position in the electro -magnetic spectrum. For example, $\mathrm{THz}$ wave has the advantage of high frequency bandwidth, low energy, high transmission and the like. So THz wave has important applications in fields like terahertz imaging, security checks, terahertz time-domain spectroscopy, $\mathrm{THz}$ radar and communications. The world gives a great deal of attention to $\mathrm{THz}$ waves just because of its wide range of applications. In recent years, state-supported $\mathrm{THz}$ development plans have been made both home and abroad. And many important achievements and progresses have been made. International scientific communities have come to an agreement that the research concerning THz science and technology is an important frontier of interdisciplinary subject. The research on atmospheric transmission of $\mathrm{THz}$ waves is the foundation of their space applications and has important academic significance and practical value ${ }^{[2-4]}$. This paper calculated and analyzed the extinction characteristics of four kinds of THz waves with the wavelengths respectively at $30 \mu \mathrm{m}, 100 \mu \mathrm{m}, 300 \mu \mathrm{m}$ and $500 \mu \mathrm{m}$ in fog. The conclusions in this paper are important for $\mathrm{THz}$ wave application in such fields as atmospheric transmission, communications and radar.

\section{Fog droplet size distribution}

Fog is a common atmospheric phenomenon, which is an aerosol suspending in the air near the ground composed of particles, water droplets or ice crystals. In terms of the visibility and droplet size, fog can be divided into haze, heavy fog, thick fog and mist. In terms of formation place and mechanism, fog can be divided into two categories: advection fog and radiation fog. Sea fog usually is advection fog while inland fog usually is radiation fog. When the $\mathrm{THz}$ wave propagates in the atmosphere, energy attenuation will usually be caused because of the scattering and absorption of atmospheric aerosol particles, especially that of fog particles. This attenuation is closely related with size distribution of fog droplets. And size distribution of fog particles in atmosphere is affected by the terrain, time,

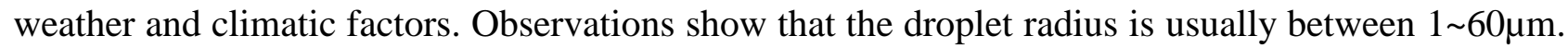
Particle size distribution is usually used to describe the distribution of fog particles in the atmosphere. And the adopted distribution is gamma distribution: 


$$
n(r)=a r^{2} \exp (-b r)
$$

Where $n$ is the number of droplets in per unit volume within per unit radius interval; $r$ is fog droplet radius. Parameters $a$ and $b$ are related with the water content $W\left(\mathrm{~g} / \mathrm{m}^{3}\right)$ and the visibility $V$ $(\mathrm{Km})$. If $r$ is in unit of $\mathrm{m}$, there are ${ }^{[5]}$

$$
a=\frac{9.781}{V^{6} W^{5}} \times 10^{15} \quad b=\frac{1.304}{V W} \times 10^{4}
$$

Visibility of fog is related with the water content. For advection fog:

$$
W=(18.035 V)^{-1.43} \quad\left(\mathrm{~g} / \mathrm{m}^{3}\right)
$$

For radiation fog:

$$
W=(42.0)^{-1.54} \quad\left(\mathrm{~g} / \mathrm{m}^{3}\right)
$$

The relationship between the two fog droplet size distribution and visibility can be obtained by combining formula (3), (4) and (1), (2). For advection fog:

$$
n(r)=1.059 \times 10^{7} V^{1.15} r^{2} \exp \left(-0.8359 V^{0.43} r\right) \quad\left(/ \mathrm{m}^{3} \cdot \mu \mathrm{m}\right)
$$

For radiation fog:

$$
n(r)=3.104 \times 10^{10} V^{1.7} r^{2} \exp \left(-4.122 V^{0.54} r\right) \quad\left(/ \mathrm{m}^{3} \cdot \mu \mathrm{m}\right)
$$

\section{The extinction parameters of group particle}

Mie scattering theory is the exact solution of isotropic homogeneous sphere electromagnetic scattering of plane. Fog droplets may be regarded as approximately equivalent of spherical particles, calculated and analyzed through Mie theory to study the fog droplets on the $\mathrm{THz}$ signal scattering and absorption.

According to Mie theory, the extinction cross section $\sigma_{t}$, scattering cross section $\sigma_{s}$ and absorption cross sections $\sigma_{a}$ of an individual particle for THz wave are respectively as follows:

$$
\begin{aligned}
& \sigma_{t}=\left(\lambda^{2} / 2 \pi\right) \sum_{n=1}^{\infty}(2 n+1)\left[\operatorname{Re}\left(a_{n}+b_{n}\right)\right] \\
& \sigma_{s}=\left(\lambda^{2} / 2 \pi\right) \sum_{n=1}^{\infty}(2 n+1)\left(\left|a_{n}\right|^{2}+\left|b_{n}\right|^{2}\right) \\
& \sigma_{a}=\sigma_{t}-\sigma_{s}
\end{aligned}
$$

Where, $\lambda$ is the wavelength, $a_{n}$ and $b_{n}$ are Mie coefficient.

$$
\begin{aligned}
& a_{n}=\frac{\varphi_{n}(\alpha) \varphi_{n}^{\prime}(m \alpha)-n \varphi_{n}^{\prime}(\alpha) \varphi_{n}(m \alpha)}{\xi_{n}(\alpha) \varphi_{n}^{\prime}(m \alpha)-n \xi_{n}^{\prime}(\alpha) \varphi_{n}(m \alpha)} \\
& b_{n}=\frac{m \varphi_{n}(\alpha) \varphi_{n}^{\prime}(m \alpha)-\varphi_{n}^{\prime}(\alpha) \varphi_{n}(m \alpha)}{m \xi_{n}(\alpha) \varphi_{n}^{\prime}(m \alpha)-\xi_{n}^{\prime}(\alpha) \varphi_{n}(m \alpha)}
\end{aligned}
$$

In Eq. (10) 


$$
\begin{aligned}
& \varphi_{n}(x)=\sqrt{\frac{x \pi}{2}} J_{n+1 / 2}(x) \\
& \xi_{n}(x)=\sqrt{\frac{x \pi}{2}} H_{n+1 / 2}^{(2)}(x)
\end{aligned}
$$

Where, $J_{n+1 / 2}(x)$ and $H_{n+1 / 2}^{(2)}(x)$ are the half-integer Bessel function and the Hankel function of the second kind; $m$ is the relative complex refractive index.

When the $\mathrm{THz}$ signal propagate in a discrete random medium, the signal energy attenuation is determined by size distribution parameters and medium extinction. total extinction coefficient $\mu_{t}$, scattering coefficient $\mu_{s}$ and absorption coefficient $\mu_{a}$ of group particle can be calculated through the following formula:

$$
\mu_{i}=\int_{0}^{\infty} \sigma_{i}(r) n(r) d r \quad(i=t, s, a)
$$

Attenuation rate (i.e., signal attenuation caused by the unit distance) can be expressed as:

$$
A=4.343 \times 10^{-3} \mu_{t} \quad(\mathrm{~dB} / \mathrm{km})
$$

\section{Calculation results and analysis}

According to the above analyses, the complex refractive index will be used in calculating fog extinction scattering parameters. Complex refractive index is an important parameter to describe the optical properties of the particle. The main ingredient of the fog droplet is water, thus the complex refractive index of fog can be replaced by the complex refractive index of water droplets. Table 1 shows the complex refractive index of fog droplets for $\mathrm{THz}$ wave at different wavelengths.

Table 1. The refractive index of fog for Terahertz wave at different wavelengths

\begin{tabular}{|c|c|c|}
\hline$\lambda / \mu \mathrm{m}$ & Real part of refractive index & Imaginary part of refractive index \\
\hline 30 & 1.551 & 0.328 \\
\hline 100 & 2.037 & 0.460 \\
\hline 300 & 2.121 & 0.490 \\
\hline 500 & 2.177 & 0.580 \\
\hline
\end{tabular}

For $\mathrm{THz}$ wave of four different wavelengths respectively at $30 \mu \mathrm{m}, 100 \mu \mathrm{m}, 300 \mu \mathrm{m}$ and $500 \mu \mathrm{m}$, according to formula (12), the relationship between scattered extinction parameters and visibility were calculated in advection fog, the results are shown in Figure 1. As can be seen, the visibility has great impact on the extinction parameters. When visibility increases, the extinction parameters will sharply decreases. This is because with the visibility increasing, the fog droplet size and concentration of particles will decrease, which will make the extinction parameters become small. Figure 1 (a) shows that the extinction is the result of joint action by scattering and absorption for the $\mathrm{THz}$ waves of $30 \mu \mathrm{m}$ atwavelength. Figure 1 (b) shows for the THz wave of $100 \mu \mathrm{m}$ wavelength, the extinction is the combined effect of scattering and absorption when the visibility is poor. When the visibility is more than $0.5 \mathrm{~km}$, extinction is mainly caused by the absorption. Figure 1 (c) (d) shows for THz waves of $300 \mu \mathrm{m}$ and $500 \mu \mathrm{m}$ wavelength, the extinctionis mainly caused by the absorption because the wavelength is much larger than the droplet size. 


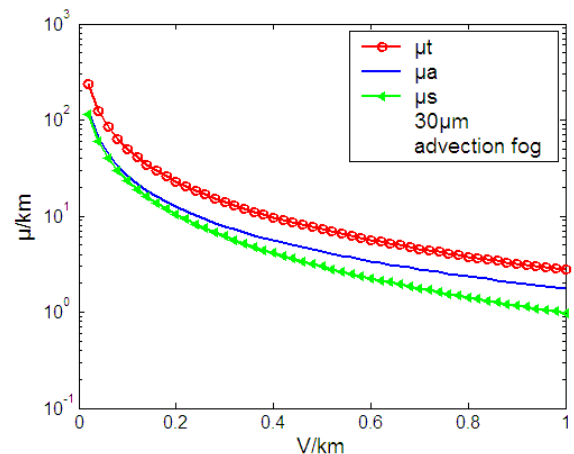

(a) $\lambda=30 \mu \mathrm{m}$

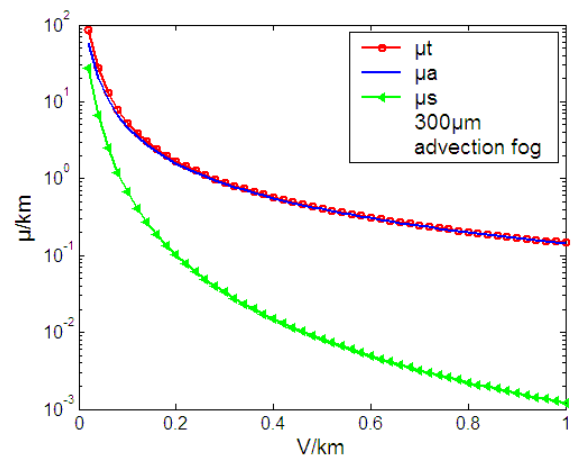

(c) $\lambda=300 \mu \mathrm{m}$

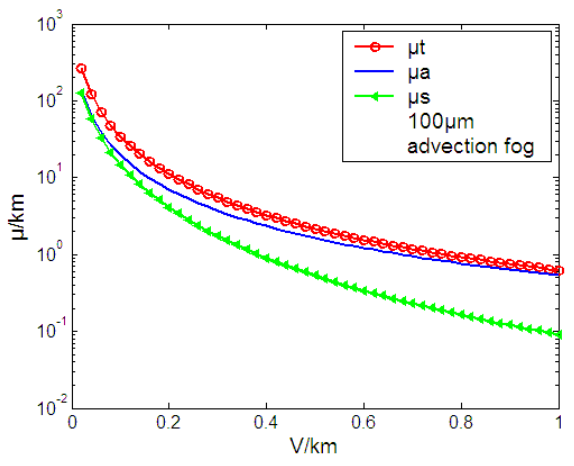

(b) $\lambda=100 \mu \mathrm{m}$

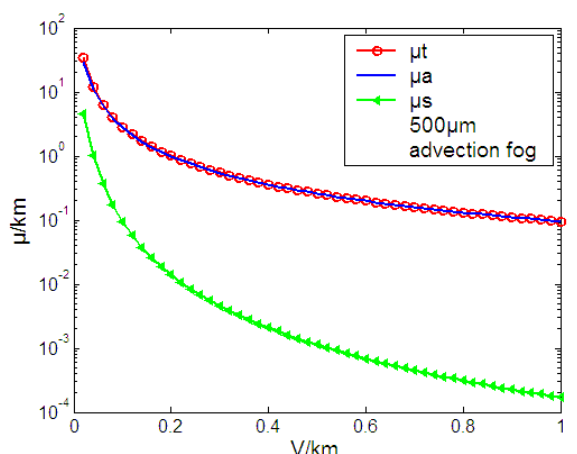

(d) $\lambda=500 \mu \mathrm{m}$

Fig.1 The relationship between extinction parameters and visibility of advection fog

Figure 2 shows the comparison between two fog extinction coefficients of $\mathrm{THz}$ waves at the same wavelength. Obviously, extinction coefficients of advection fog are bigger than those of radiation fog for the $\mathrm{THz}$ waves at four wavelengths when the visibility is the same. This is because to the droplet size of advection fog is bigger than the droplet size of the radiation fog. The difference between the extinction coefficients of the two fogs will become bigger when the visibility becomes better and better.

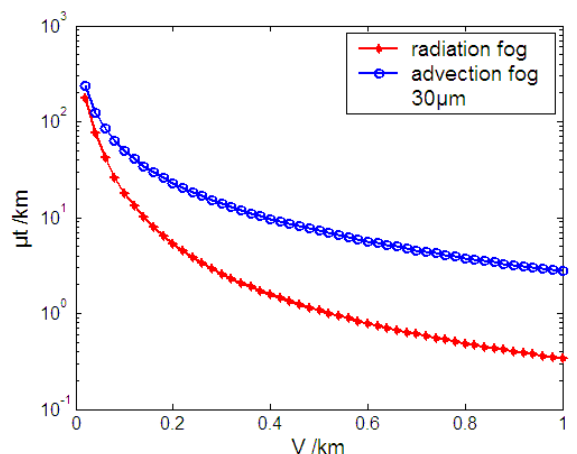

(a) $\lambda=30 \mu \mathrm{m}$

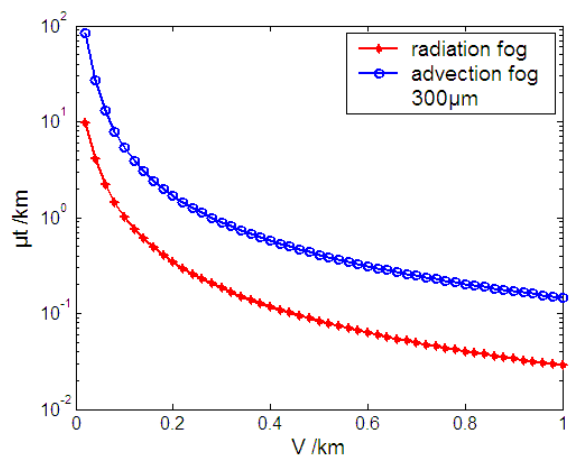

(c) $\lambda=300 \mu \mathrm{m}$

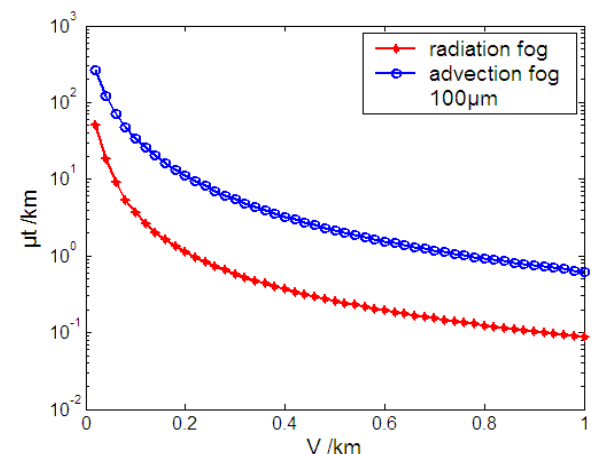

(b) $\lambda=100 \mu \mathrm{m}$

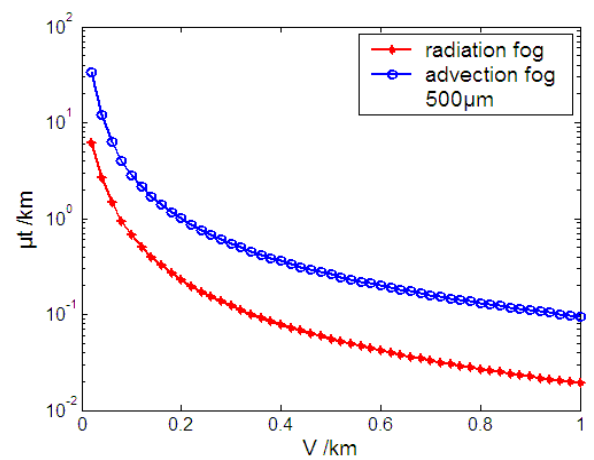

(d) $\lambda=500 \mu \mathrm{m}$

Fig. 2 The comparison of extinction coefficient of two kinds of fog 
According to equation (13), the signal attenuation caused by propagation of $\mathrm{THz}$ wave with several different wavelengths $(30 \mu \mathrm{m}, 100 \mu \mathrm{m}, 300 \mu \mathrm{m}$ and $500 \mu \mathrm{m})$ can be calculated, and the results are shown in Figure 3. It is clear that the differences of attenuation rate of fog are larger when the visibility is the same but wavelengths are different. For example, when $V$ is $0.1 \mathrm{~km}$, the THz wave attenuation rates caused by the advection fog are respectively $214.9 \mathrm{~dB}$ (for $30 \mu \mathrm{m}$ ), $149.3 \mathrm{~dB}$ (for $100 \mu \mathrm{m}$ ), $23.2 \mathrm{~dB}$ (for $300 \mu \mathrm{m}$ ) and $12.4 \mathrm{~dB}$ (for $500 \mu \mathrm{m}$ ). The longer wavelength is, the smaller the decay rate will be. It indicates that the lower the frequency of $\mathrm{THz}$ wave is, the stronger its ability to penetrate the fog will be. It is because the frequency is the lower, the difference between the wavelength and the droplet size will become the bigger, and which will weaken the extinction. Therefore, when $\mathrm{THz}$ wave is used for communication, a lower frequency $\mathrm{THz}$ wave should be the first option to consider.

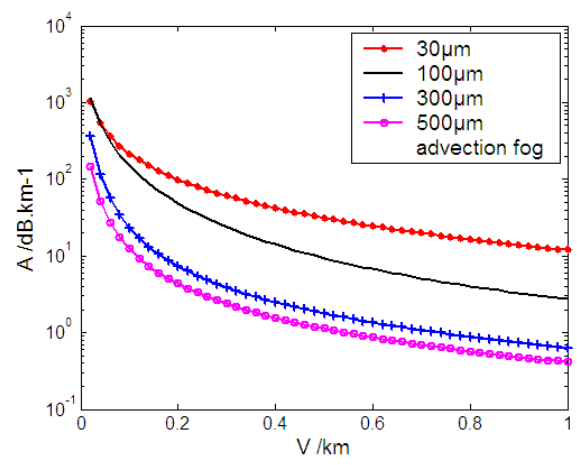

(a) Advection fog

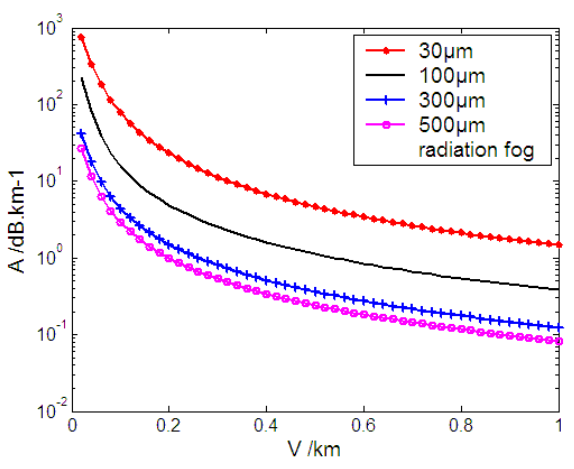

(b) Radiation fog

Fig.3 The relationship between attenuation rate and visibility of two kinds of fog

\section{Summary}

According to Mie theory and the particle size distribution of fog droplets, the total extinction of group particle were analyzed, and some important conclusions were obtained. The extinction coefficients are closely related with the visibility of the atmosphere, the kinds of fogs and the wavelength of $\mathrm{THz}$ wave. For $\mathrm{THz}$ waves at different wavelengths, when the visibility increases, the extinction coefficient will decrease sharply because of the decreasing fog droplet size. For THz wave of short wavelength, extinction is jointly caused by the absorption and scattering of fog medium. For $\mathrm{THz}$ wave of long wavelength, the extinction is mainly due to absorption. Advection fog has greater impact on the extinction of $\mathrm{THz}$ wave than radiation fog. The lower the frequency of $\mathrm{THz}$ wave is, the stronger ability it will have to penetrate the fog.

\section{References}

[1] Albert Redo Sanchez, Xi Cheng Zhang, Terahertz science and technology trends, IEEE J Sel Topics Quantun Electron. 14(2008) 60-269

[2] Kasai Yasuko, Seta Takamasa, Atmospheric propagation model of terhertz wave, Journal of the National Institute of Information and Communications Technology. 55(2008) 73-77

[3] Jana Mendrok, Philippe Baron,Yasuko Kasai, Studying the potential of terahertz radiation for deriving ice cloud microphysical information, Proc of SPIE.2008, (2008) 4-8

[4] Radoslaw Piesiewicz, Christian Jansen, Daniel Mittleman, et al, Scattering analysis for the modeling of THz communication systems, IEEE Trans Antennas Propag. 55(2007) 3002-3009

[5] Zhao Zhenwei, Wu Zhensen, Millimeter-wave Attenuation Due to Fog and Clouds, Journal of Infrared and Millimeter Waves. 20(2000) 1607-1615 